\title{
Analysis of a Pediatric Home Mechanical Ventilator Population
}

\author{
Rambod Amirnovin MD, Sara Aghamohammadi MD, Carley Riley MD, Marlyn S Woo MD, \\ and Sylvia Del Castillo MD
}

\begin{abstract}
BACKGROUND: The population of children requiring home mechanical ventilation has evolved over the years and has grown to include a variety of diagnoses and needs that have led to changes in the care of this unique population. The purpose of this study was to provide a descriptive analysis of pediatric patients requiring home mechanical ventilation after hospitalization and how the evolution of this technology has impacted their care. METHODS: A retrospective, observational, longitudinal analysis of 164 children enrolled in a university-affiliated home mechanical ventilation program over 26 years was performed. Data included each child's primary diagnosis, date of tracheostomy placement, duration of mechanical ventilation during hospitalization that consisted of home mechanical ventilator initiation, total length of pediatric ICU stay, ventilator settings at time of discharge from pediatric ICU, and disposition (home, facility, or died). Univariate, bivariate, and regression analysis was used as appropriate. RESULTS: The most common diagnosis requiring the use of home mechanical ventilation was neuromuscular disease $(53 \%)$, followed by chronic pulmonary disease $(29 \%)$. The median length of stay in the pediatric ICU decreased significantly after the implementation of a ventilator ward (70 d [30-142] vs $36 \mathrm{~d}[18-67], P=.02)$. The distribution of subjects upon discharge was home (71\%), skilled nursing facility (24\%), and died (4\%), with an increase in the proportion of subjects discharged on PEEP and those going to nursing facilities over time $(P=0.02)$. CONCLUSIONS: The evolution of home mechanical ventilation has allowed earlier transition out of the pediatric ICU and with increasing disposition to skilled nursing facilities over time. There has also been a change in ventilator management, including increased use of PEEP upon discharge, possibly driven by changes in ventilators and in-patient practice patterns. Key words: home mechanical ventilation; pediatric intensive care unit; chronic respiratory failure. [Respir Care 2018;63(5):558-564. (C) 2018 Daedalus Enterprises]
\end{abstract}

\section{Introduction}

The evolution of home mechanical ventilation over the past 30 years has influenced the care and survival of med-

\footnotetext{
Drs Amirnovin and del Castillo are affiliated with the Department of Anesthesiology Critical Care Medicine, Children's Hospital Los Angeles, Keck School of Medicine of the University of Southern California, Los Angeles. Dr Aghamohammadi is affiliated with the Division of Pediatric Critical Care Medicine, University of California Davis, Sacramento. Dr Riley is affiliated with the Division of Critical Care Medicine, Cincinnati Children's Hospital Medical Center, Cincinnati, Ohio. Dr Woo is affiliated with the Division of Pediatric Pulmonology and Sleep Medicine, University of California Los Angeles Mattel Children's Hospital, UCLA David Geffen School of Medicine, Los Angeles.
}

Dr Aghamohammadi presented a version of this report as an abstract at the Society of Critical Care Medicine Annual Congress, held January 19-23, 2013, in San Juan, Puerto Rico. ically complex pediatric patients. The population of children placed on home ventilators has not only grown substantially but has evolved to include various diagnoses and modes of ventilation to serve the particular needs of children requiring chronic ventilation ${ }^{1-6}$

The growing number of patients receiving home mechanical ventilation and the limited number of pediatric ICU (PICU) beds to provide ventilator support for stable

\footnotetext{
The authors have disclosed no conflicts of interest.

Correspondence: Rambod Amirnovin MD, Department of Anesthesiology Critical Care Medicine, Children's Hospital Los Angeles, 4650 Sunset Blvd, MS \#3, Los Angeles, CA 90027. E-mail: ramirnovin@chla.usc.edu.
}

DOI: $10.4187 /$ respcare. 05644 
patients on home mechanical ventilation prompted the creation of an alternative unit at our institution to accommodate this cohort. ${ }^{7}$ One study determined that hospitalizing subjects on home mechanical ventilation in non-ICU settings could safely be accomplished under the supervision of pediatric pulmonologists. ${ }^{7}$ Furthermore, in 1992, the amount of money saved by hospitalizing patients on a ventilator ward as opposed to an ICU was as much as $\$ 1,500$ per day. ${ }^{8}$ At our institution, a ventilator ward for non-critically ill patients on home mechanical ventilation who required hospitalization was implemented in 1993 under the guidance of the division of pediatric pulmonology.

As the home mechanical ventilation population progressed to include patients with more complex medical conditions, there has been an increased focus on their longterm outcomes after discharge from the PICU. ${ }^{9}$ The evolution of home care programs and the use of resources for children receiving chronic home mechanical ventilation either related to neuromuscular weakness or pulmonary disease has been studied. ${ }^{6,10-12}$ Results appear to show that, despite an overall higher mortality rate (up to $32 \%$ ) in patients whose disease carried a poor prognosis, chronic mechanically ventilated children could be successfully managed at home with a good home care program. ${ }^{13} \mathrm{~A}$ population analysis of chronic mechanically ventilated children in Massachusetts revealed that patients received their home care from multiple sources but lacked overall coordination of their health care providers, thereby suggesting an increasing need for improvement in health care coordination for this growing, complex population. ${ }^{1}$

There are limited studies on the pediatric home mechanical ventilation population with a paucity of patients evaluated, time-course of study, and population analysis of home mechanical ventilation patients. The body of evidence summarizing the diagnosis of pediatric patients requiring home mechanical ventilation, their disposition, and trends in the home mechanical ventilation settings used is growing but remains limited. ${ }^{14-17}$ We sought to provide a descriptive analysis of the pediatric home mechanical ventilation population at a tertiary care center in southern California serving a large population of children with chronic ventilator needs since 1977. Furthermore, we evaluated how the use of home mechanical ventilator has evolved in terms of disposition and settings over 3 decades.

\section{Methods}

\section{Data Collection}

We performed a retrospective chart review of all patients on home mechanical ventilation who received fullor part-time chronic ventilation via tracheostomy between

\section{QUICK LOOK}

\section{Current knowledge}

The increased use of home mechanical ventilators in pediatric patients since their advent in the 1970s has allowed pediatric patients to be discharged home or to long-term facilities. Understanding how this technology has changed our management of patients with chronic respiratory failure is an important step in better caring for this population.

\section{What this paper contributes to our knowledge}

With the advent of home mechanical ventilators, over 26 years of study, pediatric patients have been discharged from the ICU earlier, and more pediatric patients have been discharged to long-term facilities (versus home) and on settings more consistent with in-patient practices in respiratory care. The effects that an underlying diagnosis for respiratory failure has on disposition and ventilator settings are explored.

November 1977 and December 2008. The institutional review board at Children's Hospital, Los Angeles approved this study. Data were extracted from paper charts, the PICU database (Microsoft Access, Microsoft, Redmond, Washington), and the hospital electronic medical record system (Cerner, Kansas City, Missouri).

Subjects were included if they received tracheostomies during their admission at Children's Hospital, Los Angeles and were then initiated on home mechanical ventilation, or if they were admitted with tracheostomies but required initiation of home mechanical ventilation during their admission. We excluded patients who were admitted to the PICU with home mechanical ventilation previously established, patients only requiring supplemental oxygen via tracheostomy collar, or patients with insufficient data.

Data collected included each subject's primary diagnosis that resulted in chronic respiratory failure, the date of tracheostomy placement, the date of home mechanical ventilation initiation, the total length of PICU stay, the ventilator settings at time of discharge from PICU, and disposition upon discharge. Disposition was defined as the subject's discharge status at the end of the hospitalization within which home mechanical ventilation was initiated and was characterized as home, outside facility (sub-acute facility or referring hospital), or death.

Outcomes measured were total length of stay in the PICU, days in the PICU prior to and after tracheostomy placement (for those receiving a tracheostomy during that admission), and disposition upon discharge. The ventilator settings at the time of discharge, transfer, or death were 
obtained. $\mathrm{F}_{\mathrm{IO}_{2}}$ represents the subject's $\mathrm{F}_{\mathrm{IO}_{2}}$ prior to switching to a home ventilator, at which point flow was used to approximate the same $\mathrm{F}_{\mathrm{IO}_{2}}$. Patients discharged from our facility on home mechanical ventilation are sent home on pressure ventilation modes, with the peak inspiratory pressure implying the set peak inspiratory pressure.

\section{Data Analysis}

All variables were tested for normal distribution using a skew-kurtosis test and graphic analysis, demonstrating that all variables were non-parametric. Non-parametric continuous data were presented as medians and interquartile ranges (IQRs). Univariate analysis of patient characteristics and measured outcomes were tested with Wilcoxon rank-sum test, Fisher's exact test, and Pearson's $\chi^{2}$ tests. Linear regression analysis of parametric and parametrically transformed data were used in a step-wise fashion to find if an independent association existed between year of discharge, demographic data, individual ventilator settings, PICU lengths of stay before and after tracheostomy, and total PICU stay. Spearman's correlation was used to determine correlation coefficients and significance of association between year of discharge and PICU length of stay (including before and after tracheostomy), PEEP, and percent of patients discharged to a skilled nursing facility. Logistical regression was used to determine if changes in disposition had occurred over time (year of discharge) compared with the baseline outcome of "home." Statistical significance was defined as $P<.05$. Statistical analyses were performed with Stata (StataCorp, College Station, Texas).

\section{Results}

A total of 405 patients with tracheostomies were screened for the study; 215 did not meet inclusion criteria because their home mechanical ventilation was already established prior to admission $(n=14)$ or they were only on supplemental oxygen via tracheostomy collar $(n=201) ; 26$ were excluded for insufficient data. The remaining 164 subjects comprised our study population on full- or part-time home mechanical ventilation via tracheostomy from 1982-2008. Of these, 116 subjects (71\%) had received a tracheostomy and were transitioned to home mechanical ventilation during their PICU admission. Ninety-seven subjects (59\%) were male. The median age was 1.6 y (IQR $0.5-8.35$ y). Table 1 details the distribution of subjects by diagnosis and disposition. While many subjects had multi-factorial etiologies requiring the use of home mechanical ventilation, the most common diagnosis was neuromuscular disease (53\%), followed by chronic pulmonary disease (29\%). In terms of disposition, the vast majority of subjects were discharged home $(71 \%)$, followed by skilled nursing fa-
Table 1. Demographic Data

\begin{tabular}{lc}
\hline \hline Median age, $y$ & 1.6 \\
Female, $n(\%)$ & $67(41)$ \\
Primary diagnosis, $n(\%)$ & $87(53)$ \\
$\quad$ Neuromuscular disease & $48(29)$ \\
Pulmonary disease & $4(2)$ \\
Otolaryngology disease & $8(5)$ \\
Oncologic & $9(5)$ \\
Cardiac & $6(4)$ \\
Metabolic/Genetic & $2(1)$ \\
Miscellaneous & \\
Secondary diagnosis, $n()$ & $3(17)$ \\
Neuromuscular disease & $4(24)$ \\
Pulmonary disease & $4(24)$ \\
Otolaryngology disease & $3(17)$ \\
Cardiac & $3(17)$ \\
Metabolic/Genetic & \\
\hline$N=164$ & \\
\hline
\end{tabular}

Table 2. Distribution of Disposition Over Time

\begin{tabular}{lcc}
\hline \hline Disposition & $n(\%)$ & $P$ \\
\hline Home & $117(71)$ & Regression baseline \\
Facility & $40(24)$ & .02 \\
Died & $6(4)$ & .67 \\
Unknown & $1(1)$ & .76 \\
\cline { 1 - 1 }$N=164$ & & \\
Stepwise logistical regression analysis of distribution of disposition over time when compared \\
to the disposition of home.
\end{tabular}

cility (24\%) and death (4\%) (Table 2). The proportion of subjects with a particular diagnosis before 1993 and from 1993 on did not differ $(P=.24)$.

\section{Ventilator Settings}

Table 3 describes the ventilator settings of the subjects upon disposition from the hospital. The median breathing frequency was 23 breaths/min (IQR 16-28), peak inspiratory pressure was $28 \mathrm{~cm} \mathrm{H}_{2} \mathrm{O}$ (IQR 0-24), and PEEP was $0 \mathrm{~cm} \mathrm{H} \mathrm{H}_{2} \mathrm{O}$ (IQR 0-4). The median $\mathrm{F}_{\mathrm{IO}_{2}}$ was 0.28 (IQR 0.21-0.35). Subjects receiving PEEP had an $\mathrm{F}_{\mathrm{IO}_{2}}$ of 0.35 (IQR 0.24-0.4) versus an $\mathrm{F}_{\mathrm{IO}_{2}}$ of 0.25 (IQR 0.21-0.3) for those without PEEP $(P<.001)$. Only 2 subjects were on PEEP upon discharge before 1993, even though the discharge $\mathrm{F}_{\mathrm{IO}_{2}}$ before 1993 and from 1993 on were not different $(0.30$ [IQR $0.21-0.35$ ] vs 0.26 [IQR $0.21-0.30$ ], $P=.33$ ). The use of PEEP was higher in subjects with a primary diagnosis of chronic pulmonary disease versus those with neuromuscular disease (35\% vs $15 \%, P=.006$ ). The need for supplemental $\mathrm{F}_{\mathrm{IO}_{2}}$ was also higher in subjects 
Table 3. Ventilator Settings and PICU Length of Stay

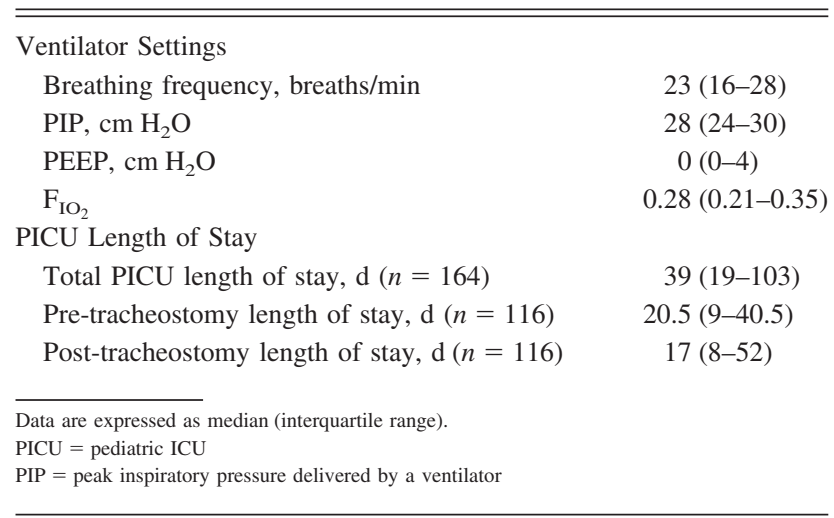

with a primary diagnosis of chronic pulmonary disease versus those with neuromuscular disease ( $83 \%$ vs $49 \%$, $P<.001)$.

\section{Outcomes}

The outcomes measured were total length of stay in the PICU, days in the PICU prior to and after tracheostomy placement for subjects who received a tracheostomy during their PICU course, and disposition upon discharge. The median total stay in the PICU for all subjects was $39 \mathrm{~d}$ (IQR 19-103). In the subset of subjects who received a tracheostomy during their hospitalization, the median total number of days in the PICU prior to tracheostomy placement was 20.5 (IQR 9-40.5) and after tracheostomy placement was 17 (IQR 8-52) (Table 3).

A ventilator ward for stable patients who were initiated and stabilized on home mechanical ventilation was established in 1993. Table 4 shows that the total length of stay in the PICU was significantly longer before the implementation of a ventilator ward compared to after it was established (70 d [IQR 30-142] vs $36 \mathrm{~d}$ [IQR 18-67], $P=.02$ ). The median length of stay in the PICU before tracheostomy was similar before and after the ventilator ward (17 $\mathrm{d}$ [IQR 10-37] vs $21 \mathrm{~d}$ [IQR 9-41], $P=.51$ ). In contrast, the median length of stay in the PICU after tracheostomy placement was higher before the implementation of a ventilator ward (39 d [IQR 21-120] vs 14 d [IQR 7-45], $P=.007)$ (Table 4).

Trends in disposition, use of PEEP, and days in the PICU after tracheostomy over the course of the 26-y study period are illustrated in Figure 1. The time periods were divided to include at least 25 subjects within each group. Over time, there was an overall decrease in the stay in the PICU after tracheostomy $(\rho=-0.38, P<.001)$, and an increase in the percentage of subjects on PEEP $(\rho=0.57$, $P<.001)$ and the percentage of subjects sent to sub-acute facilities $(\rho=0.19, P=.01)$ (Figs. 1 and 2). The temporal change in disposition shown in Figure 2 is also described in Table 2. Compared to a presumed baseline disposition of home, there was an increase in the proportion of subjects discharged to a sub-acute facility over time $(P=.02$, logistical regression). In addition, Table 5 and Figure 3 demonstrate that PEEP use was different based upon the subject's eventual disposition $(P<.001)$. When only comparing disposition to home versus a long-term facility, approximately $20 \%$ of subjects discharged home were sent on PEEP, while nearly $40 \%$ of those discharged to a subacute facility were sent while on PEEP $(P=.02)$. In contrast, the need for supplemental oxygen was not different based upon disposition $(P=.52)$, even if comparing only home versus long-term facility $(P=.35)$.

Multivariate linear regression of the parametrically transformed variables demonstrated that younger age was found to be significantly associated with longer total and posttracheostomy stay in the PICU $(P<.001)$. Additionally, this analysis confirmed that there was a decreased stay in the PICU after tracheostomy placement over the 26-y period $(P<.001)$. There was not a significant association noted between stay in the PICU and ventilator settings including breathing frequency, peak inspiratory pressure, and PEEP.

\section{Discussion}

Since its advent in 1977, the home mechanical ventilator has evolved with changes in technology, changes in practice, and the growing complexity of the patient population. A Canadian study summarized trends in a longterm mechanically ventilated pediatric population over a 20-y study period. ${ }^{11}$ They hypothesized that a combination of improvements in equipment, more aggressive care in children who would have previously died, as well as growing understanding of the family-centered benefits of living at home has led to increased home mechanical ventilation of this population. In 2010, Edwards et al ${ }^{18}$ examined outcomes and causes of death in children with home mechanical ventilation, finding that $61 \%$ of their study population survived and $18 \%$ were able to be weaned from ventilator support. Many of the deaths were unexpected and were from causes not directly related to their primary reason for chronic respiratory failure. ${ }^{18}$ Our study demonstrated a $4 \%$ in-hospital mortality rate of all subjects transitioned to a home mechanical ventilator. These studies support the findings of our study, (ie, medically complex patients supported on home mechanical ventilation are increasing in numbers) and highlight the importance of understanding this population.

Mallory and Stillwell ${ }^{19}$ suggest that younger patients with chronic pulmonary disease may require a hospital setting for long-term care, while patients with neuromuscular or central respiratory disorders may benefit from 
Table 4. PICU Length of Stay Before and After Initiation of a Ventilator Ward

\begin{tabular}{|c|c|c|c|}
\hline & Before Ventilator Ward & After Ventilator Ward & $P$ \\
\hline Total PICU length of stay, $\mathrm{d}(n=164)$ & $70(30-142)$ & $36(18-67)$ & .02 \\
\hline Pre-tracheostomy length of stay, $\mathrm{d}(n=116)$ & $17(10-37)$ & $21(9-41)$ & .51 \\
\hline Post-tracheostomy length of stay, $\mathrm{d}(n=116)$ & $39(21-120)$ & $14(7-45)$ & .007 \\
\hline
\end{tabular}

Data are expressed as median (interquartile range). The ventilator ward was implemented in 1993.

$\mathrm{PICU}=$ pediatric ICU

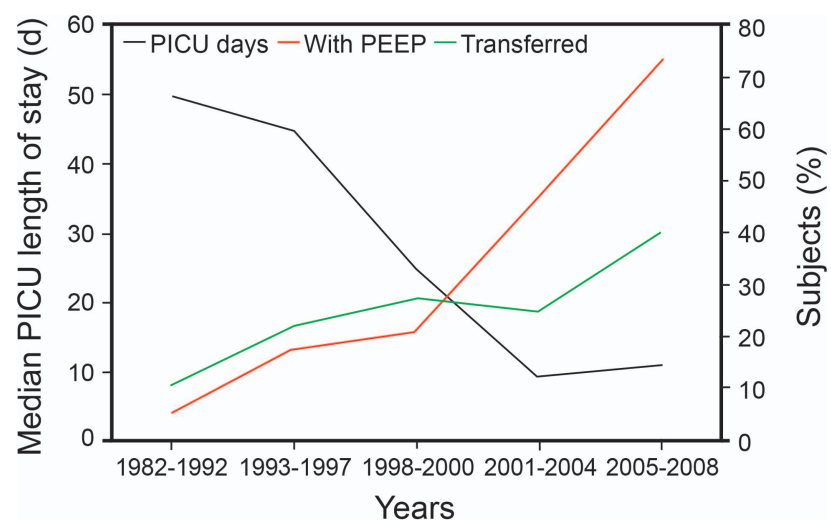

Fig. 1. Trends over time in PICU length of stay after tracheostomy ( $\rho=-0.38, P<.001)$, percent use of PEEP $(\rho=0.57, P<.001)$, and percent of subjects transferred to a long-term facility $(\rho=$ $0.19, P=.01$ ). Minimum $n=25$ per time interval.

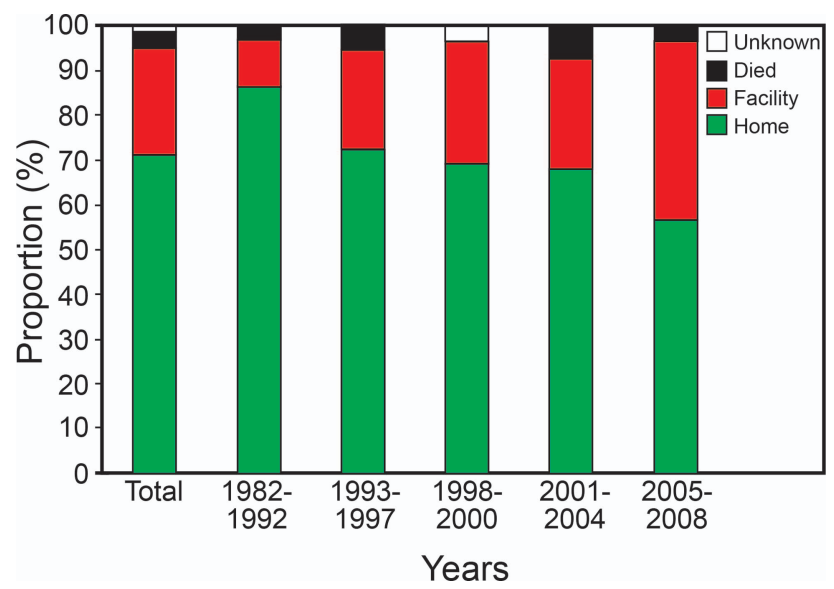

Fig. 2. Distribution of disposition over time. Data expressed as percentage of total subjects per time interval $(P=.02$, disposition to facility compared to home over time).

discharge to home. ${ }^{19,20}$ While our study demonstrated that subjects who were younger had a longer PICU length of stay, the eventual disposition did not appear to differ by the primary diagnosis alone. However, our data demonstrated that the use of PEEP and the need for supplemental $\mathrm{F}_{\mathrm{IO}_{2}}$ were associated, and subjects with chronic respiratory disease received more supplemental $\mathrm{F}_{\mathrm{IO}_{2}}$ and PEEP. Furthermore, PEEP use and discharge to a long-term facility were associated with each other and increased over time, even though supplemental $\mathrm{F}_{\mathrm{IO}_{2}}$ did not. As such, it would seem that an increased availability of home ventilators with PEEP in the 1990s, combined with the increased availability of long-term facilities, might have allowed the use of both of these in subjects who previously were sent home with supplemental oxygen alone. While the diagnosis was not related to disposition overall, it was associated with PEEP use and oxygen supplementation. These patterns likely reflect a combination of patient-related factors (eg, diagnosis and need for oxygen supplementation), technological changes (eg, available home-ventilator PEEP), and resource availability (eg, increasing long-term facilities), some of which changed over time, while others did not. The finding that neither the proportion of diagnoses nor the need for supplemental oxygen changed over time bolsters the hypothesis that the increased use of PEEP reflected changes in technology and management practices. The complex interaction between relatively static patient factors and more dynamic external factors are notable here. The finding that subjects with chronic respiratory disease received higher $\mathrm{F}_{\mathrm{IO}_{2}}$ and PEEP, while not surprising, highlights how patient diagnosis, availability of resources, and ventilator support needs should be considered when advising families of patients considering home mechanical ventilation. For example, Edwards et $\mathrm{al}^{21} \mathrm{dem}-$ onstrated the importance of considering patient factors by demonstrating the poor outcomes seen with home mechanical ventilation in subjects with congenital heart disease.

It is worth noting that the time period when the use of PEEP increased (Fig. 1) was concordant with the ARDS Network recommendations in the late 1990s regarding the use of PEEP in patients with ARDS. Furthermore, the advent of internal PEEP in home ventilators during this same time may have further accelerated the use of PEEP. It is unclear how much technology alone, versus the use of in-patient PEEP, instigated the availability of home ventilators capable of PEEP use. However, multivariable regression shows that the set PEEP did not appear to correlate with the length of PICU stay over that time. While the length of PICU stay did improve since 1993 compared to before, this may have been confounded by the creation of an acute-care ventilator ward. The increased use of PEEP 
Table 5. Disposition and Use of PEEP

\begin{tabular}{lcc}
\hline \hline \multicolumn{1}{c}{ Disposition } & With PEEP, $n(\%)$ & Without PEEP, $n(\%)$ \\
\hline Home $(n=117)$ & $25(21)$ & $92(79)$ \\
Facility $(n=40)$ & $16(40)$ & $24(60)$ \\
Died $(n=6)$ & $6(100)$ & 0 \\
Unknown $(n=1)$ & 0 & $1(100)$ \\
& \\
PEEP use differs based on eventual subject disposition $(P<.001)$. PEEP use was greater in \\
those going to a facility than in those going home $(P=.02)$.
\end{tabular}

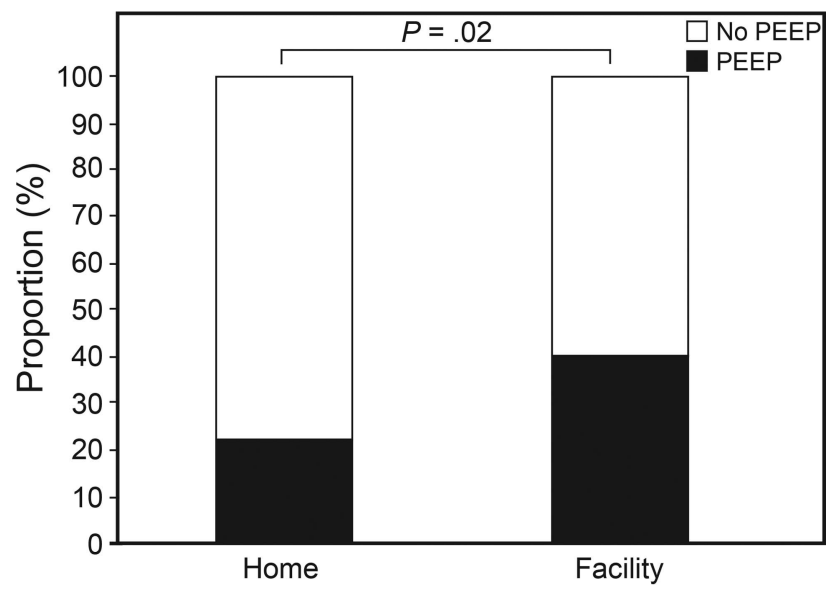

Fig. 3. Distribution of PEEP use by disposition to home or skilled nursing facility.

may suggest that changes in acute management of patients with respiratory disease can impact long-term chronic respiratory management of patients, including those disposed on home mechanical ventilation. The benefit of such transmitted management styles for chronically ventilated patients is unclear and is worthy of investigation. ${ }^{22-25}$

Our study also demonstrated that more children have been discharged to sub-acute facilities over the past 26 years. It's likely that an increased number of pediatric sub-acute facilities became available during this time. In southern California, 5 pediatric sub-acute facilities serve the Los Angeles, Orange, and San Bernadino counties. One facility was established as early as 1984, while the others were established in the late 1990s and 2000s. The increase in the proportion of children on home mechanical ventilation disposed to such facilities appears temporally related to the creation of these facilities. These facilities not only provide skilled nursing care to this high-risk population but can also provide respite care and rehabilitation activities for the children and families to participate in together. ${ }^{19,26-28}$ Arguably, the creation of such facilities has made it more plausible for patients with higher ventilator settings who may be less appropriate for home disposition to leave the hospital setting. The discovery that higher settings were used in those subjects going to such facilities may support this hypothesis. Additionally, the technology advancements in home mechanical ventilators include more portable and compact machines that allow for customized ventilator settings, such as the use of PEEP. This may have provided greater ease for patients to maintain their mobility, families to provide home care for their children, and children to benefit from more tailored ventilator settings for their disease process. ${ }^{19,27}$

Ambrosio et $\mathrm{al}^{7}$ demonstrated that chronic ventilatordependent children could be safely cared for in a non-ICU setting. This allowed for a more tailored plan of care for this unique subset of patients and provided a period of transition for patients and families to adjust to the needs of their ventilator-dependent child. Furthermore, there is a substantial difference in hospital costs between ICU and non-ICU settings as well as increasing availability of ICU beds for more critically ill children. ${ }^{7}$ Our study demonstrates that there was an overall decrease in the length of stay in the PICU over this 26-y period and that the implementation of a ventilator ward was highly associated with this trend. In 1993, the advent of a ventilator ward at our institution was associated with a decreased length of stay in the PICU after tracheostomy placement by nearly 3-fold.

\section{Limitations and Future Directions}

Our study is limited by its retrospective design. The number of subjects to analyze is inconsistent from year to year, with more subjects in later years in the investigation period. In addition, the data available were limited, including demographic data and lack of index for severity of illness. Furthermore, long-term outcome data such as morbidity and mortality based on diagnosis and disposition were not available. The addition of this information may play an important role in guiding clinicians and advising families regarding the future of their ventilator-dependent child.

\section{Conclusions}

The population of children requiring home mechanical ventilation has grown in number and complexity since the advent of the home mechanical ventilator nearly 40 years ago. The creation of a home mechanical ventilator ward augmented a shorter ICU length of stay over the years, and the increasing availability of skilled nursing facilities seems to have increased disposition to such facilities over time. Changes in home mechanical ventilation technology and clinical practice may have contributed to the increasing use of PEEP in subjects discharged on home mechanical ventilation. However, the translation of acute-care ventilator management methods to those with chronic respiratory failure is of unclear benefit. Such observational analyses are a first step in better understanding this growing population of pediatric patients. 


\section{Pediatric Home Mechanical Ventilation}

\section{ACKNOWLEDGMENTS}

We thank Dr Thomas Keens for allowing us to study the unique home ventilator population within the Pulmonology Department at Children's Hospital Los Angeles. We also thank Dr Murphy-Chutorian, who performed data collection for this study, and Tyler Nakamura for his statistical support.

\section{REFERENCES}

1. Graham RJ, Fleegler EW, Robinson WM. Chronic ventilator need in the community: A 2005 pediatric census of Massachusetts. Pediatrics 2007;119(6):1280-1287.

2. Eigenn H, Zander J. Home mechanical ventilation of pediatric patients. Am J Respir Crit Care Med 1990;141(1):258-259.

3. Graf JM, Montagnino BA, Hueckel R, McPherson ML. Pediatric tracheostomies: a recent experience from one academic center. Pediatric Crit Care Med 2008;9(1):96.

4. Ottonello G, Ferrari I, Pirroddi IM, Diana MC, Villa G, Nahum L, et al. Home mechanical ventilation in children: Retrospective survey of a pediatric population. Pediatrics International 2007;49(6):801805.

5. Schreiner MS, Donar ME, Kettrick RG. Pediatric home mechanical ventilation. Pediatr Clin North Am 1987;34(1):47-60.

6. Sritippayawan S, Kun SS, Keens TG, Davidson SL. Initiation of home mechanical ventilation in children with neuromuscular diseases. J Pediatr 2003;142(5):481-485.

7. Ambrosio IU, Woo MS, Jansen MT, Keens TG. Safety of hospitalized ventilator-dependent children outside of the intensive care unit. Pediatrics 1998;101(2):257-259.

8. Edwards JD, Rivanis C, Kun SS, Caughey AB, Keens TG. Costs of hospitalized ventilator-dependent children: differences between a ventilator ward and intensive care unit. Pediatr Pulmonol 2011;46(4): 356-361.

9. Yates K, Festa M, Gillis J, Waters K, North K. Outcome of children with neuromuscular disease admitted to paediatric intensive care. Arch Dis Child 2004;89(2):170-175.

10. Sakakihara Y, Yamanaka T, Kajii M, Kamoshita S. Long-term ventilator-assisted children in japan: a national survey. Acta Paediatr Jpn 1996;38(2):137-142.

11. Amin R, Sayal P, Syed F, Chaves A, Moraes TJ, Maclusky I. Pediatric long-term home mechanical ventilation: twenty years of follow-up from one Canadian center. Pediatr Pulmonol 2014;49(8): 816-824.

12. Briassoulis G, Filippou O, Natsi L, Mavrikiou M, Hatzis T. Acute and chronic paediatric intensive care patients: current trends and perspectives on resource utilization. QJM 2004;97(8):507-518.
13. Canlas-Yamsuan M, Sanchez I, Kesselman M, Chernick V. Morbidity and mortality patterns of ventilator-dependent children in a home care program. Clin Pediatr 1993;32(12):706-713.

14. Amin RS, Fitton CM. Tracheostomy and home ventilation in children. Semin Neonatol 2003;8(2):127-135.

15. Edwards EA, Hsiao K, Nixon GM. Paediatric home ventilatory support: the Auckland experience. J Paediatr Child Health 2005;41(12): 652-658.

16. Paulides FM, Plotz FB, Verweij-van den Oudenrijn LP, van Gestel JP, Kampelmacher MJ. Thirty years of home mechanical ventilation in children: escalating need for pediatric intensive care beds. Intensive Care Med 2012;38(5):847-852.

17. Tibballs J, Henning R, Robertson CF, Massie J, Hochmann M, Carter $\mathrm{B}$, et al. A home respiratory support programme for children by parents and layperson carers. J Paediatr Child Health 2010;46(1-2): 57-62.

18. Edwards JD, Kun SS, Keens TG. Outcomes and causes of death in children on home mechanical ventilation via tracheostomy: an institutional and literature review. J Pediatr 2010;157(6):955-959.

19. Mallory GB, Jr, Stillwell PC. The ventilator-dependent child: issues in diagnosis and management. Arch Phys Med Rehabil 1991;72(1): 43-55.

20. Millner BN. Technology-dependent children in New York state. Bull N Y Acad Med 1991;67(2):131-142.

21. Edwards JD, Kun SS, Keens TG, Khemani RG, Moromisato DY. Children with corrected or palliated congenital heart disease on home mechanical ventilation. Pediatr Pulmonol 2010;45(7):645-649.

22. Boroughs D, Dougherty JA. Care of technology-dependent children in the home. Home Health Nurse 2009;27(1):37-42.

23. Boroughs D, Dougherty JA. Decreasing accidental mortality of ventilator-dependent children at home: a call to action. Home Healthc Nurse 2012;30(2):103-111.

24. Murphy J. Medically stable children in PICU: better at home. Paediatr Nurs 2008;20(1):14-16.

25. DeWitt P, Jansen M, Ward S, Keens T. Obstacles to discharge of ventilator-assisted children from the hospital to home. Chest 1993; 103(5):1560-1565.

26. Allen NL, Simone JA, Wingenbach GF. Families with a ventilatorassisted child: transitional issues. J Perinatol 1994;14(1):48-55.

27. Warner J, Norwood S. Psychosocial concerns of the ventilator-dependent child in the pediatric intensive care unit. AACN Clin Issues Crit Care Nurs 1991;2(3):432-445.

28. Farrell PM, Fost NC. Long-term mechanical ventilation in pediatric respiratory failure: medical and ethical considerations. Am J Respir Crit Care Med 1989;140(2 Pt 2):S36-S40. 